\title{
EXPRESSION OF INSULIN LIKE GROWTH FACTOR IN THE CEREBROSPINAL FLUID OF CHILDREN WITH HYDROCEPHALUS
}

\author{
F. Mashayekhi, Z. Salehi \\ Department of Cell and Molecular Biology, Islamic Azad University, Tonekabon Branch, Tonekabon, Iran
}

\begin{abstract}
Insulin like growth factor-1 (IGF-1) is ubiquitously expressed growth factor that has profound effects on the survival, growth and differentiation of many cell types and tissues, including cells of the central nervous system (CNS). IGF-1 is found in many biological fluids including cerebrospinal fluid (CSF). IGF-1 has broad range neuroprotective effects and is a therapeutic candidate for neurodegenerative diseases. Hydrocephalus is a condition in which there is an abnormal build-up of CSF within the ventricles and/or subarachnoid spaces. Since CSF is in contact with the extracellular space of the brain, biochemical brain modifications could be reflected in the CSF. The aim of this study was to determine the level of CSF IGF-1 level in the children with non-communicating hydrocephalus. A total of 46 samples of CSF from normal and children with hydrocephalic were collected by lumbar puncture performed routinely on the basis of the clinical suspicion of neurological disease. Samples were aged matched between the two groups analyzed. A western blot analysis using anti-IGF-1 antibody as a probe confirmed the presence of IGF-1 in the samples. Quantification of Western blot gels showed that the amount of IGF-1 was increased in the hydrocephalus CSF when compared with normal controls. Using ELISA, it was shown that the concentration of CSF IGF-1 in the hydrocephalus was significantly higher than in controls. The data from this study indicate that IGF-1 is a constant component of human CSF. It is also concluded that high levels of CSF IGF-1 may be partly related to hydrocephalus pathophysiology.
\end{abstract}

